\title{
Les instruments transitionnels : une proposition pour étudier la diachronie des activités narratives
}

Transitional instruments: a proposal for studying narrative activity in a diachronic way

\section{Anne Bationo-Tillon, Viviane Folcher et Pierre Rabardel}

\section{OpenEdition}

\section{Journals}

Édition électronique

URL : http://journals.openedition.org/activites/2437

DOI : 10.4000/activites. 2437

ISSN : 1765-2723

Éditeur

ARPACT - Association Recherches et Pratiques sur les ACTivités

\section{Référence électronique}

Anne Bationo-Tillon, Viviane Folcher et Pierre Rabardel, « Les instruments transitionnels : une proposition pour étudier la diachronie des activités narratives », Activités [En ligne], 7-2 | octobre 2010 mis en ligne le 15 octobre 2010, consulté le 21 avril 2019. URL : http://journals.openedition.org/ activites/2437 ; DOI : 10.4000/activites.2437

\section{c) (i) $\Theta$}

Activités est mis à disposition selon les termes de la licence Creative Commons Attribution - Pas d'Utilisation Commerciale - Pas de Modification 4.0 International. 


\title{
Les instruments transitionnels : \\ une proposition pour étudier la diachronie des activités narratives
}

\section{Anne Bationo-Tillon, Viviane Folcher et Pierre Rabardel}

\author{
Université Paris 8/laboratoire Paragraphe/équipe C3U \\ 2 rue de la liberté, 93526 Saint Denis cedex \\ anne.bationo-tillon@univ-paris8.fr \\ Viviane.Folcher@univ-paris8.fr \\ Pierre.Rabardel@univ-paris8.fr
}

\begin{abstract}
Transitional instruments: a proposal for studying narrative activity in a diachronic way. This paper presents the narrative activity of two polar explorers. We have adopted a unit of diachronic analysis that encompasses the collection procedure during the journey, as well as the drafting of the story afterwards. With this in mind, we focus on two theoretical frameworks: the instrumental approach and the course of action. We suggest using the concept of "transitional instrument" to identify what is occurring within these discontinuous narrative activities. During the journey, the narrators work with various instruments (e.g. photographs, notes) as a way of distancing themselves from the experience. At this stage they create their own transitional instruments. After the journey, the narrators are once again immersed in the travel experience through a display of the items collected, such as sketches, notes and maps. They compose the story by assembling the transitional instruments. We will conclude with a discussion of how diachronicity contributes to a better understanding of creative activities and the related tools.
\end{abstract}

\section{KEYWORDS}

narrative activities, creative activities, diachronic activities, instrumental approach, course of action.

\section{1.- Les activités narratives et les outils d'aide à la narration}

La réflexion autour des outils d'aide aux narrations collectives dans un cadre de communauté de pratiques (Barriquault, 2005 ; Soulier, 2006) connaît un regain. Dans ces situations, le processus de narration peut être collectif et conduit progressivement à un diagnostic contextuel, localisé et partagé de la situation (0rr, 1996). Dans le domaine de la vie quotidienne, les outils de narration et de présentation de soi sont largement investis par le grand public même s'ils n'ont qu'une vie parfois éphémère comme les pages personnelles (Beaudouin, \& Velkovska, 1999). Dans un contexte de fort développement des technologies de l'information et de la communication, ils se transforment et sont rapidement remplacés par d'autres tels que les blogues (Cardon, \& Delaunay-Teterel, 2006), et plus récemment tous les réseaux sociaux permettant d'échanger et exposer son réseau tout en se racontant au quotidien (facebook, twitter, etc.). Cependant, d'autres études montrent que malgré la profusion de nouvelles technologies disponibles, les pratiques de collage, d'albums photos et de journaux herbiers ancrées dans le monde physique subsistent (Lejeune, 1998 ; West, Quigley, \& Kay, 2007). Cette combinaison du monde réel et numérique a d'ailleurs été largement investiguée par les 
chercheurs en IHM via la conception d'outils d'aide à la narration principalement dans le champ de l'éducation (Ackerman, 2008 ; Agnosti, De Michelis, Divitini, Grasso, \& Snowdon, 2002 ; Decortis, Daele, Polazzi, Rizzo \& Saudelli, 2001 ; Glos, \& Cassel, 1997 ; Umaschi, 1996). A l'exception de Decortis Daele, Polazzi, Rizzo et Saudelli (2001), ces outils sont pensés pour supporter la narration mais ils ne tiennent pas compte des différents espaces et temps de la narration. Nous nous focalisons sur des activités individuelles multi-instrumentées « en train de se faire», qui se déroulent dans le temps et l'espace.

Dans cet article nous allons étudier et comprendre des activités narratives créatives en situation naturelle, nous qualifions ces activités narratives « d'activités productives diachroniques » dans la mesure où elles sont orientées vers la réalisation d'une tâche au long cours, de l'ordre de la mission. Dans un premier temps, nous expliciterons le contexte de la recherche et les partis pris qui ont été adoptés pour étudier ces activités. Dans un second temps, nous exposerons le cadre théorique et nous définirons les instruments transitionnels, concept introduit dans cette étude pour rendre compte des activités narratives discontinues. Nous décrirons ensuite les méthodologies mobilisées pour examiner les activités narratives de deux expéditeurs polaires. Quelques résultats seront présentés afin d'étayer les apports de la diachronie. Nous terminerons par une discussion autour des contributions de cette recherche au plan épistémique et pragmatique.

\section{2.- Les activités narratives : multi-instrumentation et diachronie}

Comme bon nombre d'activités créatives, les activités narratives n'ont pas de solutions et de chemins prédéterminés et s'inscrivent dans des temps longs. À ce titre, elles se rapprochent du travail des architectes (Lebahar, 1983) d'une part, car la prescription est « floue » et d'autre part, car ce sont des activités qui laissent une grande place aux aléas de la subjectivité. Lebahar (1983) évoque l'absence de conventions dans la simulation graphique des architectes propre à celui qui la produit, elle est son style, lisible seulement pour et par lui-même. Il explique que comprendre les activités des architectes réside dans l'explicitation du rapport entre ce qui se déroule dans la tête de l'architecte et le dessin en train de se construire. Il rappelle que l'activité créative est plus riche que ce que le dessin laisse apparaître. À notre sens, comprendre les activités narratives consiste également à établir des ponts entre les traces de la narration en train de se faire et ce qui est significatif pour le narrateur. Abordons maintenant le double ancrage industriel et théorique de notre étude.

\section{1.- De l'ancrage industriel aux activités narratives multi-instrumentées}

Ce travail de recherche a été réalisé dans le cadre d'un doctorat d'ergonomie financé par une entreprise de télécommunication en mutation (Bationo-Tillon, 2006). A l'époque, l'entreprise ambitionnait d'élargir son cœur de métier en fournissant non seulement les différents réseaux (téléphonie mobile, internet, téléphonie fixe) mais également des services associés à ces trois types de réseaux autour des contenus numériques (par exemple : photographies, vidéo, blogues, documents numériques, musique, etc.). La demande était donc située en amont de la conception de services d'aide aux activités narratives, elle n'avait pas pour vocation d'alimenter un projet spécifique mais plusieurs projets de conception $^{1}$ très hétérogènes en termes de techniques, de cibles, de fonctionnalités et de contextes d'utilisation. Il s'agissait donc d'un travail de prospective dont les résultats pouvaient ensuite être adressés à des interlocuteurs variés au sein de l'entreprise (marketing, informaticiens, concepteurs d'IHM, concepteurs de services, etc.) pour instruire des choix d'orientation au sein de différents

1. Des projets très variés qui questionnent la mobilité tels que la conception de service d'aide aux touristes, la reconception d'un site internet de partage de photos de voyage, la conception de stylo et papiers communicants, la conception d'un logiciel de reconnaissance d'écriture, etc. Ces projets concernent des cibles très hétéroclites (du touriste au consultant professionnel) (en déplacement, à domicile, en voyage), des technologies plus ou moins matures (du site internet partageable classique au stylo communicant). On entend par stylo communicant, un stylo classique augmenté de propriétés numériques, ce type de dispositif s'inscrit dans la lignée des objets communicants qui visent à allier les avantages du monde physique et du monde digital. 
projets. L'occasion nous était donc donnée par ce travail de prospective d'effectuer une intervention ergonomique la plus en amont possible d'avant le projet. Dans ce contexte, nous cherchions à comprendre comment concevoir des outils qui ne limitent pas la création, comment concevoir des outils qui supportent les diverses formes des activités narratives créatives.

Nous délimitons notre angle d'étude aux activités narratives multi-instrumentées. Nous traitons les récits d'évènements vécus et nous incluons tous les artefacts (photographie, vidéo, dessin, écrit, etc.) pouvant s'articuler au cours de la production d'un récit. Nous avons choisi d'aborder les activités narratives en situation naturelle de manière holistique en adoptant une unité d'analyse large que nous qualifions de diachronique. Nous nous sommes focalisés sur les activités narratives de recueil au cours d'un déplacement (prise de photographie, prise de notes, croquis, etc.) suivies des activités narratives de mise en forme des éléments recueillis (rédaction d'un récit). Nous avons donc considéré ce positionnement en amont comme une occasion d'étudier la diversité des activités instrumentées par le biais de la diachronie.

\section{2.- De la diachronie à l'ancrage théorique}

Les activités narratives sont discontinues et flexibles dans la mesure où les différents matériaux de la narration se construisent en strates au cours du temps. Ces matériaux sont constitués puis sélectionnés, agencés, réarticulés pour produire un récit. A notre sens, les activités narratives permettent à l'individu de relier le passé et le futur, de se replacer dans une continuité, une logique critique afin de renouer avec le sens. La diachronie est donc un prisme intéressant pour approcher la volatilité et les temps successifs de ces activités narratives discontinues. Pour rendre compte du cheminement diachronique des activités narratives, le cours d'action (Theureau, 2004) et l'approche instrumentale (Rabardel, 1995) ont été mobilisés de façon complémentaire. Ces deux approches nous permettent d'effectuer des aller-retours entre une description et compréhension singulière et générique des activités narratives mais également d'explorer leurs différentes temporalités (mailles synchronique et diachronique). Par ailleurs, elles se rejoignent sur l'importance qu'elles donnent au subjectif, point central à nos yeux pour étudier les activités narratives.

Le cours d'action nous a permis de rendre compte du caractère temporel continu ou discontinu de ces activités narratives. Il offre la possibilité de maintenir les différentes histoires concomitantes aux activités narratives dans lesquelles le narrateur est engagé, ceci est possible uniquement en restant au plus près du cours d'expérience singulier. Ainsi le cours d'action permet de retravailler, redécouper le temps chronologique pour constituer des unités significatives élémentaires (use) de l'acteur. Ces uses correspondent aux actions, interprétations, focalisations, émotions ou communications de l'acteur. Elles émergent grâce à la description qui articule le point de vue de l'observateur chercheur et le point de vue propre de l'acteur.

L'approche instrumentale quant à elle nous a permis d'étudier et de spécifier la nature des médiations des situations instrumentées, mais aussi de nous intéresser à l'instrument en fonction du sens que le sujet lui attribue, et non de l'outil catégorisé d'un point de vue technocentré. En effet, pour Rabardel l'instrument est une entité mixte qui tient à la fois de l'artefact et du sujet. Plus précisément, l'instrument est constitué de deux composantes : un artefact et un schème (Piaget, 1974 ; Vergnaud, 1985). Le schème d'après Piaget est une organisation active de l'expérience vécue qui intègre le passé. Vergnaud a prolongé cette définition en proposant de relier les caractéristiques opératoires des situations aux schèmes. Selon lui le schème est constitué de quatre composantes que sont les buts, les règles, les invariants opératoires et les inférences. Rabardel s'inspire conjointement de la notion de schème ainsi que des théories de l'activité (Vygostky, 1934) pour rendre compte des médiations de l'activité. L'instrument constitue un médiateur dans le rapport du sujet à lui-même (médiation réflexive), à autrui (médiation interpersonnelle) et à l'objet de son activité. Ainsi, par ses concepts de systèmes d'instruments, classes de situation, et schèmes d'utilisation, l'approche instrumentale était un cadre d'analyse nous permettant d'extraire des situations d'activité instrumentée (Rabardel, 2001). 
En résumé, le cours d'action permet de saisir les questions de temps et l'approche instrumentale intègre l'analyse des instruments. D'un point de vue méthodologique, nous avons utilisé le cours d'action dans un premier temps pour établir une trame temporelle des activités étudiées, puis dans un second temps, nous avons effectué à partir de cette description, une extraction des situations d'activité instrumentée.

\section{3.- Que nous révèle la diachronie des activités narratives ?}

Les activités narratives provoquent une situation paradoxale qui exige des narrateurs qu'ils sortent de la situation dans laquelle ils sont immergés ici et maintenant. Lors du déplacement, ils s'efforcent de s'extraire momentanément de l'expérience en train de se faire pour effectuer le recueil, en revanche lors de l'élaboration du récit, après le déplacement, ils s'efforcent de se reconnecter avec l'expérience du déplacement pour élaborer le récit. La diachronie nous a amenés à adopter une unité d'analyse large qui englobe la constitution du recueil lors du déplacement ainsi que l'élaboration du récit après le déplacement (voir figure 1).

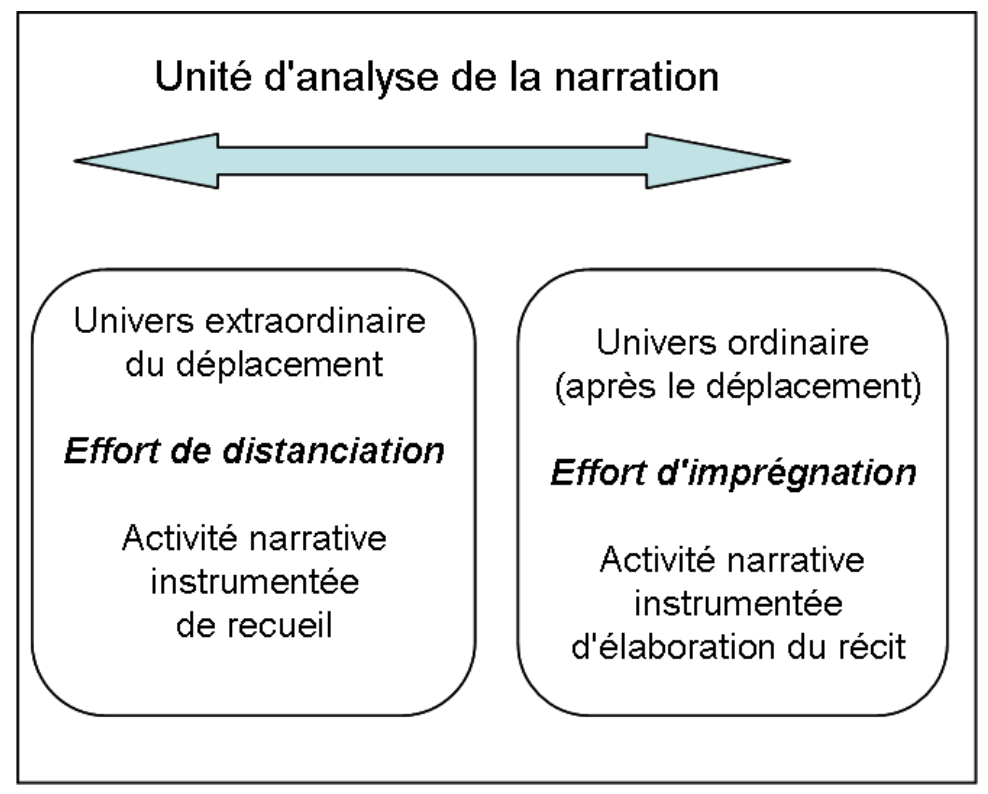

Figure 1: Les activités narratives diachroniques

Figure 1: Diachronic narrative activities

\section{1.- Une mise à distance de l'expérience}

Bruner (1990) rappelle que la narration nous permet de donner un sens à nos expériences et de les partager avec les autres. Dans cette optique, le récit consiste à raconter des choses survenues dans notre quotidien, il perpétue l'expérience dans la mémoire.

Decortis, Daele, Polazzi, Rizzo et Saudelli (2001) se sont inspirés de la pensée vygostkienne et de son cycle de l'imagination (1973) pour étudier les activités narratives d'enfants dans le cadre du projet POGO. Selon Vygotky, l'activité créative de notre imagination dépend de la richesse et de la variété de nos expériences. Le modèle POGO rend compte de l'ancrage de ces activités narratives dans l'expérience quotidienne. Il y aurait tout d'abord l'exploration qui correspondrait à l'interaction avec le monde réel dans toutes ses dimensions sensorielles, l'inspiration (choix des idées à développer), la production (sélection et association d'éléments), puis le partage. Lors d'un déplacement, les activités narratives viennent s'immiscer, bousculer l'expérience en train de se faire pour tenter de découper et fixer le flux de l'expérience. Barthes (2003) souligne la tension entre deux attitudes qui coexistent 
chez l'écrivain flâneur : remplir son carnet et explorer, vivre des expériences dans le monde, rencontrer des personnes. Nous pensons que cette tension est présente chez tous les narrateurs au moment de leurs pérégrinations qu'ils soient ethnologues, journalistes, écrivains ou passionnés de voyages. «On croit qu'on va faire un voyage, mais bientôt c'est le voyage qui vous fait ou vous défait » (Bouvier, 1992). Le voyage est donc la découverte de nouvelles contrées mais également un dialogue avec soi-même. Or sans la collecte et l'écriture, le visible resterait confus, désordonné et sombrerait dans l'oubli (Laplantine, 1996). Face au constat que « Le présent colle aux yeux » (Barthes, 2003), une manière de prendre de la distance consiste à noter le présent au fur et à mesure. Le langage permet donc de lier, séparer et fixer des contenus dans le continuum du flux. Il classe et organise l'expérience (Meyerson, 1948). Ceci ne se limite pas au langage, Bourdieu (1965) postule que la photographie est une classification volontaire du passé. En effet, le simple fait de prendre une photographie suppose une distance par rapport au présent, se nourrissant du sentiment que l'instant à retenir bascule déjà dans le passé.

Ainsi tout en s'imprégnant d'un nouvel univers, le narrateur choisit délibérément d'effectuer un recueil à l'aide d'une diversité d'outils qui vont lui permettre de prendre de la distance vis-à-vis de son expérience en train de se faire.

\section{2.- Une ré-imprégnation de l'expérience}

Les activités narratives s'inscrivent dans une succession d'étapes nécessairement distribuées dans l'espace et au cours du temps. Nous venons de décrire la percolation des activités de recueil avec les activités d'exploration d'un nouvel univers. Arrêtons-nous sur les étapes ultérieures, lorsque le narrateur transforme les éléments collectés, annotés en vue d'élaborer un récit.

Pinault (1994) souligne cette transformation en comparant le journal de voyage de Hoël (peintre, sculpteur, dessinateur) contenant des informations très hétérogènes et son récit final qui est une recréation du voyage. Latour (1996) s'est penché sur une expédition d'anthropologues au Brésil. Il met en évidence les transformations, transmutations, traductions successives effectuées par les anthropologues sur les éléments recueillis dans la savane jusqu'à leur transformation en publication scientifique. Il parle d'une chaîne de référence dans la mesure où la forêt brésilienne devient des échantillons de mottes de terres collectées, les mots échangés au cours de l'exploration de la forêt deviennent des phrases écrites dans un carnet, les couleurs observées de la terre brésilienne deviennent des chiffres notés dans un carnet. A chaque étape successive, cette chaîne réversible perd un peu de sa matérialité et de sa complexité mais gagne en transportabilité, en maniabilité et utilisation possible dans un réseau de plus en plus large, et dans des cadres de réflexions déjà existants. Cependant, pour manipuler et déplacer ces matériaux pour faire émerger des patterns, il faut revenir aux moments où la référence est encore attachée à son contexte d'origine, au milieu circonstanciel où ces matériaux s'appuient sur des désignations ostensives de ce qui est là (Dodier, 1990).

Ainsi, les éléments du récit sont transformés, modifiés, remodelés, désarticulés au cours du temps jusqu'à trouver un point d'équilibre, une cohérence. A ce titre, Bruner (1990) rappelle que l'objet d'un récit ne réside pas dans le fait qu'il colle à une quelconque réalité cachée, mais qu'il aboutisse à une cohérence, à une adéquation externe et interne.

Il nous semble donc que les questions d'imprégnation et distanciation de l'expérience se situent au cœur des activités narratives. Elles traversent le narrateur de part en part, ce dernier se trouve successivement partagé entre l'immersion dans un nouvel univers et la distanciation avec l'expérience en train de se faire via les activités de recueil d'une part, puis la nécessaire ré-imprégnation avec son expérience passée que requiert le travail d'élaboration du récit d'autre part. 


\section{4.- Des instruments transitionnels entre imprégnation et distanciation?}

Selon Winnicott (1971), l'objet transitionnel pour le nouveau-né et les phénomènes transitionnels pour l'adulte sont des relais permettant de sentir que l'expérience ne s'est pas abruptement terminée, à savoir une aire neutre d'expérience qui ne sera pas contestée. Cette aire intermédiaire d'expériences est un lieu de repos pour l'individu qui consiste à maintenir, à la fois séparées et reliées l'une à l'autre, réalité intérieure (subjective) et réalité extérieure (objective). Il souligne que ces phénomènes transitionnels marquent la progression vers l'expérience vécue. Il montre comment l'objet transitionnel est désinvesti au moment où se développent les intérêts culturels de l'enfant. Cependant, d'après lui, ces phénomènes transitionnels ne disparaissent pas mais se transforment, deviennent diffus et se répandent dans la zone intermédiaire qui subsistera tout au long de la vie de l'individu dans les arts, la vie imaginaire, le travail scientifique créatif. Selon lui, ces phénomènes transitionnels sont donc présents chez tout individu pour se libérer de la tension suscitée par la mise en relation de la réalité du dedans et de la réalité du dehors. Dans cette perspective, les phénomènes transitionnels permettent au sujet de garder le lien avec son expérience tout en l'objectivant, la regardant comme extérieure à soi. Or, les matériaux de la narration sont constitués d'éléments hétérogènes collectés, fabriqués ou capturés lors du déplacement, ils permettent de découper le flux du réel, de l'organiser. Puis dans un second temps, ils jouent le rôle de médiateurs pour le narrateur qui revisite des moments passés, s'imprègne à nouveau de l'expérience vécue pour élaborer un récit. Ils marquent la progression vers l'expérience vécue, vers la distanciation tout en étant indispensables pour la ré-imprégnation. Il s'agit donc de phénomènes transitionnels au sens de Winnicott.

Nous regroupons ces matériaux hétérogènes sous la catégorie générique d'instruments transitionnels afin de chercher à comprendre leurs propriétés génériques au sein de l'activité narrative. Nous proposons une première caractérisation :

- Tout d'abord, un instrument transitionnel est un artefact inanimé auquel le narrateur confère un certain nombre de propriétés. Les instruments transitionnels sont des artefacts constitués, fabriqués, ou appropriés. Un artefact devient instrument transitionnel à partir du moment où un sujet lui attribue un statut particulier d'aide mémoire, de témoin, de dépositaire des traces d'une expérience ou encore d'une observation effectuée.

- Un instrument est transitionnel car le sujet le fait «transiter » d'une situation (un lieu, un espace, un moment et un contexte donné) à une autre, et l'utilise dans diverses activités discontinues dans le temps, et ancrées dans des situations différentes. La finalité d'un artefact de ce type est sa réutilisation dans un autre contexte, dans un autre environnement. L'instrument transitionnel ne contient pas uniquement l'idée de médiation (déjà présente dans la définition d'instrument) mais également de transition entre une situation spatio-temporelle (s1) à t et une autre (s2) à t'. Ces artefacts transitionnels sont donc la matérialisation des liens créés par les sujets pour constituer de la cohérence, de la continuité (du point de vue intrinsèque) au sein d'activités discontinues (du point de vue extrinsèque).

- Ces artefacts transitent d'une situation à une autre tout en maintenant une certaine unité de l'individu ou de l'expérience et/ou, tout en conservant et maintenant une certaine information de la situation (s1). Au moment de sa constitution, l'instrument transitionnel permet de prendre la distance nécessaire avec l'expérience en train de se faire. Il s'agit d'un instrument de découpage du réel qui colle aux yeux du narrateur. Lors de sa réutilisation ultérieure, il permet au narrateur de se replonger dans ses impressions passées, de se reconnecter avec son expérience, avec la situation de référence.

Le concept d'instrument transitionnel est un outil théorique au sein de notre travail pour penser et étudier la diachronie au sein des activités narratives. Nous chercherons à rendre compte des propriétés des instruments transitionnels que nos études empiriques ont fait émerger, ainsi que de leur rôle 
au sein des activités narratives.

\section{5.- L'activité narrative de deux expéditeurs polaires}

Cette étude se focalise plus particulièrement sur les activités narratives d'un domaine spécifique (l'expédition polaire) dans l'objectif d'appréhender les instruments transitionnels et de décrire les activités narratives discontinues dans lesquelles ces instruments transitionnels s'insèrent. Nous avons retenu cette situation extrême dans laquelle évoluaient des expéditeurs-chercheurs, car la constitution d'un recueil de données est indispensable pour la communauté des expéditeurs, mais également pour le développement de leurs propres activités de recherche. En effet, les fortes contraintes climatiques et l'isolement obligent les expéditeurs à rendre compte de leur activité ainsi que des difficultés rencontrées au jour le jour, afin de capitaliser cette expérience pour les expéditions futures. D'autre part, leurs carnets de bord qu'ils soient écrits ou filmés, présentent un intérêt particulier puisqu'il s'agit à la fois du carnet de l'ethnologue (la sociologie au sens large constitue les domaines de recherche des deux participants chercheurs), du carnet du sportif (l'expédition polaire est une performance sportive), du journal intime (l'isolement et la durée confrontent l'expéditeur à lui-même), du carnet de voyage (avec la particularité d'un fort dépaysement), voire du retour d'expérience de situation à risque.

\section{1.- Terrain}

L'expédition polaire se compose de trois membres. Une personne expérimentée et passionnée d'expéditions polaires, dont la devise « Une année sans expédition est une année perdue » illustre la place laissée aux expéditions polaires dans sa vie. Cette personne, que nous nommerons P1 au cours de l'étude, allie ses objectifs de recherche (sociologie / gestion) et l'aventure de l'expédition polaire. Il est le chef d'expédition. Une deuxième personne que nous baptiserons P2 est ethnologue. Une troisième personne P3 fait également converger des aspects de ses recherches en sciences humaines avec les expéditions polaires. Cependant, sa discipline nécessitant moins de recueil de notes que l'ethnologie ou la sociologie, nous avons donc décidé d'analyser uniquement les activités narratives de P1 et P2.

L'expédition a duré 15 jours. Concernant les outils de recueil, P1 a à sa disposition une micro caméra pour tenir un journal de bord vidéo, deux carnets distincts, des cartes géographiques, des fiches techniques et un GPS. Quant à P2, chercheur ethnologue qui n'avait aucune expérience de l'expédition polaire et qui combine ses objets de recherche avec ce nouveau terrain, ses outils de recueil sont plus sommaires : un carnet noir, un carnet beige, un appareil photo numérique et une montre.

Les activités au cours de l'expédition consistent à ranger et plier le matériel dans les pulkas, skier, faire de la voile, se restaurer, monter et démonter le campement, préparer le repas du soir et comprennent plus précisément les activités narratives qui consistent à prendre des notes dans des carnets de bord, prendre des photos, filmer, etc. Nous nous intéresserons également aux activités narratives qui ont lieu après l'expédition: autrement dit les activités de production d'un récit à partir des éléments recueillis par les expéditeurs. Les activités narratives de ces deux expéditeurs-chercheurs polaires se sont déroulées en pointillés sur 16 semaines environ.

\section{2.- Méthodologie}

Nous avons effectué des enregistrements vidéo, d'une part pour documenter l'activité narrative dans son aspect temporel et dynamique, d'autre part pour que le chercheur ne soit pas en présence des sujets, ni même observateur des activités en temps réel. Ceci nous semblait être la meilleure solution étant donné les conditions extrêmes du terrain, et l'intimité requise de ces activités. L'enregistrement via une micro-caméra fixée sur les lunettes des participants nous a permis d'accéder aux éléments manipulés, lus, écrits par les expéditeurs. Ce système enregistrait en continu les activités de prise de 
notes pendant l'expédition ou de rédaction de ces notes au retour. Des entretiens en resitu subjectifs (Rix \& Biache, 2004) et entretiens d'autoconfrontation à partir des traces de l'activité ont été menés a posteriori. Ces entretiens ont été menés selon une démarche commune de questionnement, en insistant sur les questions autour des préoccupations, des éléments auxquels le sujet portait attention, ainsi que sur l'explicitation des actions effectuées (Theureau, 2004 ; Vermersch, 1994).

Comme le montre le Tableau 1 ci-dessous, les données recueillies étaient variées et composées de vidéo, traces des activités de narration (journal de bord vidéo, carnets, fiches, récits) ainsi que d'entretiens.

\begin{tabular}{|c|c|c|c|}
\hline \multicolumn{2}{|r|}{ Matériel } & Expéditeur 1 & Expéditeur 2 \\
\hline \multirow{2}{*}{$\mathrm{T} 2 \mathrm{xD} 2$} & Vidéo : perspective subjective & 4heures 30 & 3 heures 30 \\
\hline & Vidéo : perspective externe & 4 heures 30 & 3 heures 30 \\
\hline \multirow{3}{*}{ D4 } & Photos & 0 & 30 \\
\hline & Journal de bord écrit & 90 pages & passages \\
\hline & Journal de bord vidéo & 4 heures & $\mathrm{O}$ \\
\hline \multirow[b]{2}{*}{ T2’xD3 } & entretien en resitu subjectif (T2) & 6 heures & 4 heures \\
\hline & $\begin{array}{l}\text { Autoconfrontation à partir du } \\
\text { matériel personnel }\end{array}$ & $\begin{array}{l}5 \text { heures (journal de bord } \\
\text { vidéo, journal de notes) }\end{array}$ & $\begin{array}{l}1 \text { heures (photos+ carnet } \\
\text { beige de questions) }\end{array}$ \\
\hline \multirow[t]{2}{*}{$\mathrm{T} 3 \mathrm{xD} 2$} & $\begin{array}{l}\text { Vidéo perspective subjective } \\
\text { activité traitement des données } \\
\text { (T3) }\end{array}$ & 10 heures & 1heure 30 \\
\hline & $\begin{array}{l}\text { Vidéo perspective externe activité } \\
\text { traitement (T3) }\end{array}$ & 8 heures & $1 \mathrm{~h} 30$ \\
\hline D4 & $\begin{array}{l}\text { Versions intermédiaires, } \\
\text { Versions finales }\end{array}$ & $\begin{array}{l}1 \text { version intermédiaire } \\
1 \text { version finale }\end{array}$ & 1 version intermédiaire \\
\hline \multirow[b]{2}{*}{ T3’xD3 } & entretien en resitu subjectif (T4) & 6 heures & heures \\
\hline & $\begin{array}{l}\text { Autoconfrontation à partir du } \\
\text { matériel personnel (T4) }\end{array}$ & 2 heures & Photos : 30 minutes \\
\hline
\end{tabular}

Tableau 1 : Données recueillies tout au long de l'étude

Table 1: Data collected during the study

Il était demandé aux acteurs à T2 (durant l'expédition polaire) de prendre des notes, photos, comme ils en avaient l'habitude. Ils devaient par contre l'annoncer à P3 avant de commencer afin que P3 installe le début de l'enregistrement selon les angles, distances convenues au préalable avec le chercheur au cours du week-end de préparation. Il était demandé aux acteurs à T3 (au retour de l'expédition polaire) d'enclencher l'enregistrement audio et vidéo subjectif et externe au moment, où ils retravaillaient les données, le recueil issu de l'expédition. Il n'y avait donc aucune obligation de temps, de non interruptions ou d'interruptions. Nous cherchions à observer leurs pratiques in situ de la manière la plus naturelle possible.

Mentionnons quelques difficultés rencontrées. Le cahier de voyage / journal de bord présente un caractère très intime. En effet, le journal de bord est un espace où chaque personne évoque ses états d'âme, son impression du pays. Il peut donc parfois se rapprocher du journal intime, il s'avère donc difficile d'y accéder, tout en respectant l'intimité de chacun. Les traces du voyage sont très hétérogènes en termes de quantité et type de recueil. De plus, les personnes interrogées n'ont pas le même parcours. L'expérience pratique réelle ainsi que l'échelle temporelle de l'élaboration du récit sont différentes.

Du point de vue des analyses, la démarche a été la suivante. Après avoir transcrit les entretiens et après avoir codé les enregistrements audio et vidéo, il s'agit de constituer des récits réduits (au sens 
de Theureau, 2004), à partir de la mise en correspondance, en utilisant la complémentarité des informations issues de chaque description (objectivable et intrinsèque). C'est ce qui nous permet d'obtenir une description signifiante du point de vue de l'acteur.

Le schéma ci-dessous résume nos deux mailles d'analyses mobilisées au sein de cette étude

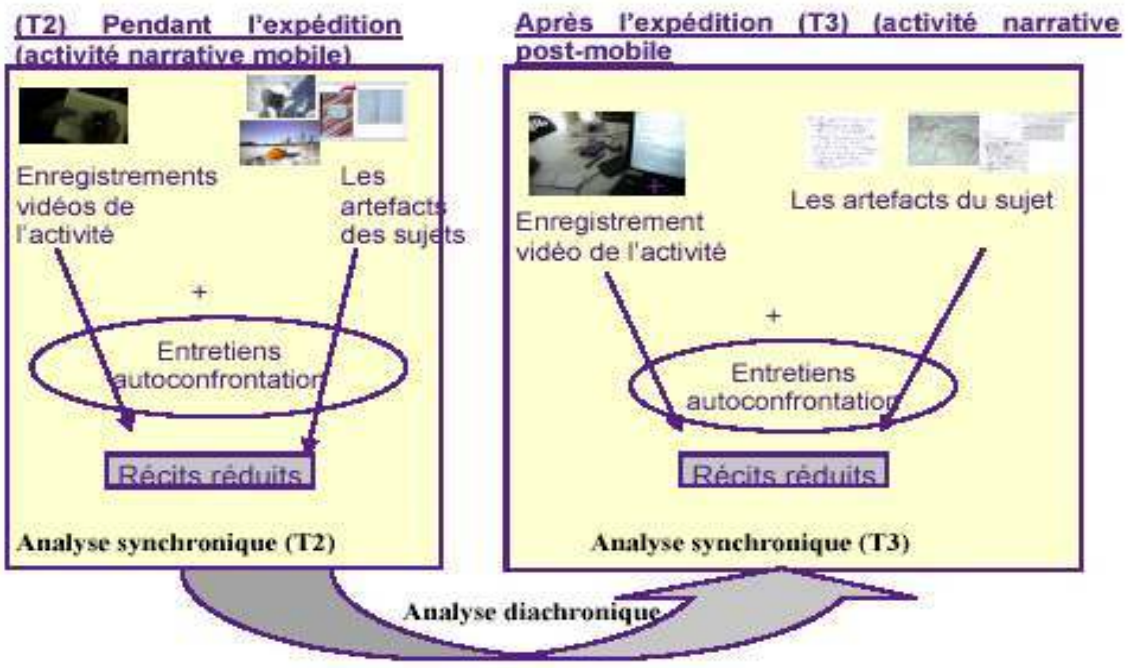

Figure 2 : Les deux unités d'analyse : synchronique et diachronique

Figure 2: The two units of analysis: synchronic and diachronic

Nous avons effectué des analyses synchroniques et diachroniques. Autrement dit, la mise en évidence de récits réduits (enchaînement des unités significatives élémentaires (uses)), de schèmes et d'instruments correspondent à ce que nous appelons une analyse synchronique, appartenant à un espace temporel confiné à T2 (expédition polaire) ou T3 (retour de l'expédition polaire). Tandis que les instruments transitionnels sont de l'ordre de l'analyse diachronique dans la mesure où ils font le lien, la jonction entre les deux espaces temporels T2 et T3.

\section{6.- Les éclairages réciproques de la synchronie et de la diachronie}

Dans un premier temps, nous spécifierons les caractéristiques des activités narratives au cours du déplacement. Dans un second temps nous présenterons un invariant de l'activité présent dans l'activité de nos narrateurs lors de l'élaboration du récit, que nous avons nommé le schème d'étalage. Nous terminerons enfin par la présentation du fonctionnement des instruments transitionnels identifiés.

\section{1.- Des instruments de recueil narratif : une mise à distance de l'expérience}

Rappelons que la constitution d'un recueil concourt à une mise à distance de l'expérience en train de se faire. En conséquence, cette activité ne peut se comprendre hors de son contexte d'origine. Si on considère les instruments de recueil comme des instruments pour découper le flux d'expérience, il existe plusieurs manières de découper ce flux d'expérience. En effet, nous avons décelé au sein de l'activité des deux expéditeurs polaires des instruments de recueil divers que nous présentons dans le tableau 2 ci-dessous. Rappelons que le découpage des instruments (Rabardel, 1995) n'est pas superposable au découpage technique des supports : un instrument de recueil descriptif peut tout autant renvoyer à un fragment d'une séquence vidéo, à une photo ou à un paragraphe inscrit dans un carnet de voyage. Chacun de ces instruments de recueil présente un type médiation spécifique à l'environnement du déplacement. 


\begin{tabular}{|c|c|c|}
\hline $\begin{array}{c}\text { Type } \\
\text { d'instruments }\end{array}$ & Préoccupations des narrateurs & $\begin{array}{l}\text { Illustration issue de l'activité d'un des deux } \\
\text { expéditeurs }\end{array}$ \\
\hline $\begin{array}{l}\text { Instrument } \\
\text { scanner }\end{array}$ & $\begin{array}{l}\text { Ramener un morceau de } \\
\text { l'expérience sensorielle de l'univers } \\
\text { de déplacement (vernaculaire, } \\
\text { auditive, sensitive, etc.) }\end{array}$ & Pas observé chez les expéditeurs polaires \\
\hline $\begin{array}{l}\text { Instrument } \\
\text { descriptif (au } \\
\text { fil de l'eau) }\end{array}$ & $\begin{array}{l}\text { Rendre compte de l'environnement, } \\
\text { rendre compte de ce qui est } \\
\text { caractéristique à ce pays : fragments } \\
\text { lisibles a posteriori selon la trame de } \\
\text { l'espace. }\end{array}$ & $\begin{array}{l}\text { Fragment du journal de bord de } \mathrm{P} 1 \text { où il décrit } \\
\text { les lieux tout en pointant à l'aide de son bâton } \\
\text { les références. }\end{array}$ \\
\hline $\begin{array}{l}\text { Instrument } \\
\text { d'explication } \\
\text { d'événements } \\
\text { (organisé) }\end{array}$ & $\begin{array}{l}\text { Rendre compte de la chronologie } \\
\text { du séjour (rendre compte des } \\
\text { évènements passés, du présent et du } \\
\text { futur). }\end{array}$ & 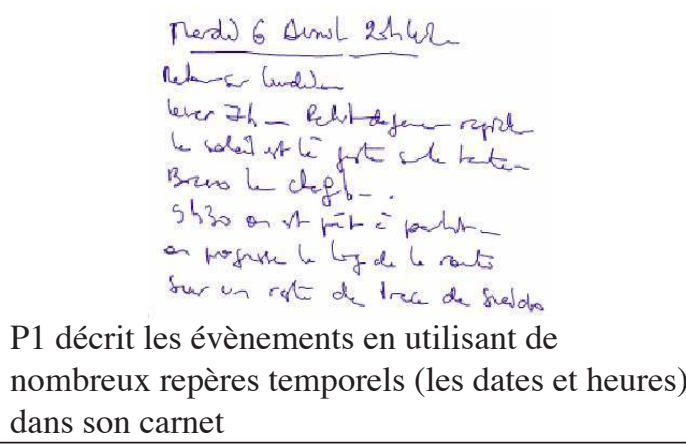 \\
\hline $\begin{array}{l}\text { Type } \\
\text { d'instruments }\end{array}$ & Préoccupations des narrateurs & Illustration pour un des deux expéditeurs \\
\hline $\begin{array}{l}\text { Instrument } \\
\text { dialogiques (fil } \\
\text { de l'eau) }\end{array}$ & $\begin{array}{l}\text { Garder une trace de réflexions } \\
\text { personnelles, techniques, de } \\
\text { recherche. } \\
\text { Garder une trace de ses réflexions, } \\
\text { de ses sensations ou connaissances } \\
\text { en résonnance avec l'expérience en } \\
\text { train de se faire. }\end{array}$ & $\begin{array}{l}\text { P1 précise le besoin impérieux de noter ses } \\
\text { réflexions dans son carnet au moment où elles } \\
\text { viennent sous forme de liste, pour les avoir } \\
\text { ensuite, à sa disposition. }\end{array}$ \\
\hline $\begin{array}{l}\text { Instruments de } \\
\text { documentation } \\
\text { d'intérêts } \\
\text { (organisé) }\end{array}$ & $\begin{array}{l}\text { Rationaliser le recueil en } \\
\text { documentant pour chaque } \\
\text { phénomène des catégories, des } \\
\text { matrices souvent définies a priori. }\end{array}$ & $\begin{array}{l}\text { Fiches de P2 qui sont conçues de manière à } \\
\text { renseigner les catégories suivantes pour chaque } \\
\text { phénomène observé ( } \mathrm{N}^{\circ} \text { de fiche, thème, lieu, } \\
\text { date, observations, commentaires). }\end{array}$ \\
\hline
\end{tabular}

Tableau 2 :Typlogie des instruments de recueil

Table 2: Typology of collection instruments 
Au travers des données, nous mettons en évidence six types de médiation à l'environnement du déplacement :

Le schème scanner consiste à collecter, cueillir des éléments, des copeaux de l'univers de déplacement quasiment bruts.

Les schèmes de narration descriptifs consistent à constituer des fragments permettant de conserver des éléments selon une logique topographique de l'univers du déplacement.

Les schèmes de narration d'explication d'évènements forment des fragments préservant des éléments de l'expérience de déplacement selon une logique temporelle.

Les schèmes de documentation d'intérêts participent à la composition de fragments permettant d'enregistrer des commentaires, des explications relatives à un phénomène rencontré lors du déplacement tout en respectant une logique de complétude et d'exhaustivité,

Les schèmes de narration dialogique façonnent des fragments qui permettent de maintenir les impressions subjectives, les réflexions du narrateur au cours de son déplacement.

Les schèmes d'énonciation permettent de plus ou moins accentuer la logique chronologique, topographique, complémentaire, voire l'assemblage de toutes ces logiques au sein des activités narratives. De plus, nous pensons que c'est bien grâce à la diversité des médiations des instruments de recueil que les narrateurs se retrouvent dans la possibilité de rationnaliser, mettre à distance l'information qu'ils doivent ensuite réélaborer pour produire un récit.

A partir de cette typologie des instruments de recueil, notons qu'il est possible d'effectuer une distinction supplémentaire : les instruments de recueil dialogique correspondent à une externalisation et une réification des sentiments, des pensées du narrateur lors du voyage tandis que les matériaux issus des instruments descriptifs, de documentation et chronologiques ont trait au découpage, à la transcription, à la fixation des éléments perçus du voyage. Ces dernières seraient donc le fruit d'un mouvement d'internalisation du narrateur en train de s'approprier l'univers du déplacement. Ainsi deux mouvements coexisteraient lors des activités de recueil : d'une part l'internalisation de la manière dont le narrateur comprend et découpe le monde qu'il parcourt et d'autre part l'externalisation de ses pensées, ressentis surgissant lors de sa découverte de ce monde. Il résulte de cette tension un recueil constitué de matériaux hétérogènes, qui se retrouvent consignés au même niveau quelles que soient leurs genèses, c'est à notre sens ce qui permet au narrateur de ne pas évincer sa subjectivité au regard des matériaux plus «mesurables » et de pouvoir effectuer des choix pour élaborer le récit.

\section{2.- le schème d'étalage multi-instrumenté: une ré-imprégnation de l'expérience}

Au retour du déplacement, pour élaborer son récit, le narrateur organise son recueil afin d'en faciliter la consultation puis il se constitue un espace de travail au sein duquel il produira son récit. Il effectue ensuite un va et vient entre la consultation du recueil et la production du récit. C'est cet aller-retour qui lui permet de replonger dans le voyage et qui se manifeste sous la forme d'un schème d'étalage multi-instrumenté. Nous présentons d'abord les supports de ce schème d'étalage que sont les espaces de consultation et de production. Dans un second temps, nous décrivons son fonctionnement.

\section{L'espace de consultation}

Ce schème d'étalage multi-instrumenté repose en partie sur l'espace de consultation. Au moment où le narrateur rentre de son déplacement et se retrouve à son domicile ou dans son bureau, il met à plat tout son recueil, en l'étalant. Ceci peut se matérialiser par des tas de documents jonchant le sol et le bureau, par l'affichage de cartes et de documents sur le mur, par la projection de diapositives et vidéo. Cet étalage permet de rendre à la fois visibles, lisibles et concomitants tous les éléments recueillis au cours du déplacement. Le narrateur peut s'immerger dans le recueil effectué au cours 
du déplacement en le manipulant, en le feuilletant et en naviguant à l'intérieur de chaque média (cahier, vidéo, photos, etc.) ainsi que de manière transverse aux différents supports. Ceci n'est possible qu'à partir du moment où il dispose dans un même espace spatio-temporel les éléments collectés et élaborés à divers moments et endroits de l'univers du déplacement. Précisons, que des documents supplémentaires au recueil peuvent être ajoutés tels qu'une encyclopédie, des cartes géographiques, des journaux de bords d'autres expéditeurs polaires, etc.

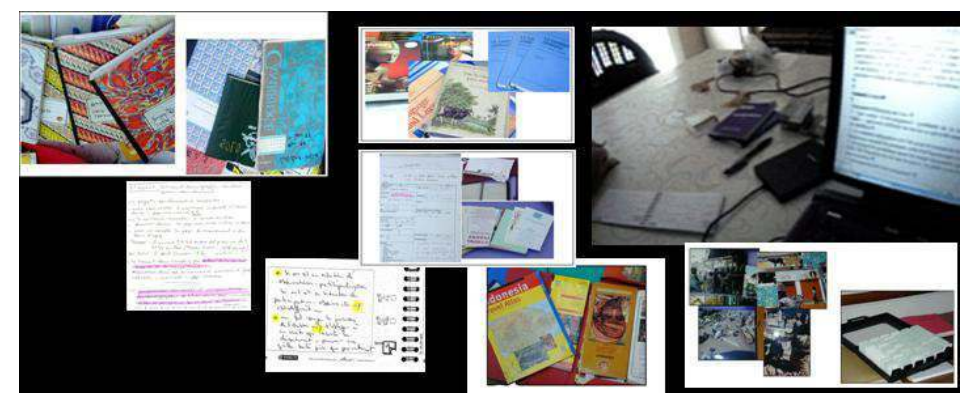

Figure 3 : L'étalage de la diversité des artefacts

Figure 3: The display of the various artefacts

\section{L'espace de production}

Ce schème d'étalage multi-instrumenté se situe à l'articulation de l'espace de consultation et de l'espace de production, dans la mesure où l'espace de consultation rend à la fois visibles, lisibles et concomitants tous les éléments recueillis au cours du déplacement, et du même coup possible l'élaboration du récit en permettant au narrateur de sélectionner, d'acheminer certains éléments du recueil dans l'espace de production, d'en mettre d'autres de côté, de synthétiser certaines informations et d'en développer d'autres. La manière de sélectionner, de taire certains éléments et d'organiser son récit au sein de l'espace de production dépend de l'objet d'activité du narrateur. En effet, selon que le narrateur est engagé dans l'écriture d'une nouvelle humoristique ou d'un article scientifique, les opérations de gommage et de mise en exergue des divers registres du récit seront très différentes.

\section{Description du schème d'étalage multi-instrumenté}

Décrivons maintenant le schème d'étalage multi-instrumenté, à partir de la définition de Vergnaud (1985), tel que nous l'avons analysé au sein de l'activité d'un des expéditeurs polaires (P1). Ce dernier était engagé dans la rédaction d'un récit de retour d'expérience. En effet, à l'issue de l'expédition, en tant que chef d'expédition, il se doit de produire un journal de bord afin de capitaliser l'expérience de cette expédition pour que la communauté des expéditeurs bénéficie de l'expérience des uns et des autres.

Ce schème d'étalage s'organise tout d'abord autour d'un but principal qui est la production d'un journal de bord chronologique transmissible à l'ensemble de la communauté comme le précise l'expéditeur en entretien : «Il faut capitaliser l'expérience [...], les expéditeurs polaires ont tous une bibliothèque incroyable en terme de journal de bord, en partant des plus illustres de Pierre Emile Victor... mais aussi le journal de bord de connaissances qui sont allés au Spitzberg, un mois »

De plus, ce schème est constitué de deux sous-buts qui consistent :

- à s'imprégner du voyage en s'appuyant sur l'espace de consultation constitué de multiples instruments ;

— à sélectionner les éléments pertinents pour alimenter l'espace de production. Il s'agit d'une part de gommer des éléments jugés trop personnels, intimes « Il y a des choses qu'on va garder pour soi, certaines choses sont moins intéressantes pour les autres, ce sont plus des 
réflexions personnelles ». D'autre part, le narrateur met en exergue la description du périple, des problèmes rencontrés, des réflexions techniques relatives à l'organisation, au matériel :

« J'explique les contraintes qu'a cette nouvelle tente qui nous pose problème ».

Les invariants opératoires qui guident la prise d'information, la collecte d'information au sein de l'espace de consultation, sont principalement les points de repères chronologiques que sont les dates ou encore des points de repères autour des évènements qui permettent de retrouver la chronologie du parcours. Il re-parcourt tout son recueil hétérogène (journal de bord vidéo, divers carnets) afin de décrire le plus précisément possible le déroulement de l'expédition « Là tu rajoutes des informations dans le lundi 29 mars? Oui, je développe tous les éléments du vol en voyant les images... parce qu'il y a des éléments butoirs qui sont vraiment des faits qui te permettent de retrouver ce qui s'est passé».

Deux règles organisent la suite des actions de ce narrateur : le respect de la chronologie, un niveau de description à peu près équivalent pour chaque jour, ainsi que la description de tous les incidents. Ceci se matérialise par sa manière de construire chaque paragraphe autour d'un titre qui correspond à la date et aux évènements marquants de la journée. Ensuite au sein de chaque paragraphe, à l'aide du journal de bord vidéo et des carnets, il décrit de manière chronologique (du matin au soir) le déroulement de la journée.

Enfin le sujet met en œuvre des inférences et des anticipations autour de l'enchainement, de la lisibilité, de la cohérence de son récit afin qu'il soit partageable et lisible par les membres de la communauté des expéditeurs.

Ce schème d'étalage s'enracine donc dans l'espace de consultation pour nourrir l'espace de production, il mobilise une diversité d'instruments d'imprégnation présents au sein de l'espace de consultation et d'instruments d'édition au sein de l'espace de production. En fonction de l'objet de l'activité, la manière de tisser de la continuité entre les activités narratives antérieures (recueil présent dans l'espace de consultation) et les activités narratives ultérieures (élaboration du récit dans l'espace de travail) sera différente. Dans le cadre de la rédaction d'une nouvelle humoristique par exemple, le narrateur revisitera son recueil et son voyage sous l'angle de l'anecdote, tandis que dans le cadre de l'écriture d'un papier scientifique, le narrateur se replongera dans son voyage en étant attentif à recouper les faits. Il y a donc une part active du narrateur pour se mettre en situation d'évocation, de ré-imprégnation avec son expérience passée. En effet, lors de l'étalage, lorsque le narrateur consulte son recueil, il se retrouve dans la position du lecteur de toute narration. Or, dans tout récit, il y a des ellipses, et comme le disent Eco (1985), Bordwell (1985) et Mc Cloud (1993), le récit est un tissu de non-dit, de l'invisible, quel que soit le support. Le lecteur va combler ces vides, actualiser ce récit incomplet grâce à son expérience du monde et en fonction de l'objet de l'activité (type de récit à élaborer).

\section{3.- Des instruments transitionnels aux activités narratives polymorphes}

Le concept d'instrument transitionnel nous permet de penser dans le même temps, les activités narratives au cours du déplacement via la constitution d'un recueil et les activités narratives après le déplacement via l'élaboration du récit à partir du recueil. Le tableau ci-dessous présente les caractéristiques des principaux instruments transitionnels identifiés. Ces instruments transitionnels sont constitués via plusieurs supports (carnets, vidéo, etc.), puis ils sont repris, redécoupés et intégrés au sein d'un seul et même support (un document informatique). Ainsi, le découpage des instruments transitionnels n'est pas superposable au découpage technique des supports. 


\begin{tabular}{|c|c|c|c|c|}
\hline $\begin{array}{l}\text { Formes de } \\
\text { l'activité }\end{array}$ & $\begin{array}{c}\text { Type } \\
\text { d'instrument } \\
\text { transitionnel }\end{array}$ & $\begin{array}{l}\text { Exemples de contenus } \\
\text { enregistrés au moment } \\
\text { du recueil (au cours du } \\
\text { déplacement) }\end{array}$ & $\begin{array}{l}\text { composantes } \\
\text { de l'activité } \\
\text { du narrateur } \\
\text { au moment de } \\
\text { l'élaboration du } \\
\text { récit (après le } \\
\text { déplacement) }\end{array}$ & $\begin{array}{l}\text { Nature des } \\
\text { transformations } \\
\text { des fragments } \\
\text { de la narration }\end{array}$ \\
\hline \multirow{2}{*}{ linéaire } & Miroir & $\begin{array}{l}\text { Dates, lieux, } \\
\text { informations formelles et } \\
\text { officielles, etc. Diversité } \\
\text { des médias (écrit, } \\
\text { croquis, images, etc.) }\end{array}$ & $\begin{array}{l}\text { repris à l'identique: } \\
\text { copié, reproduit }\end{array}$ & $\begin{array}{l}\text { transformation } \\
\text { de la mise en } \\
\text { forme ou la taille } \\
\text { (police, taille } \\
\text { image, etc.) }\end{array}$ \\
\hline & Traduit & $\begin{array}{l}\text { paysages, date, } \\
\text { informations chiffrées, } \\
\text { etc. (images, écrits, etc.) }\end{array}$ & $\begin{array}{l}\text { Repris comme source } \\
\text { à transcrire : traduit } \\
\text { d'un référentiel à un } \\
\text { autre, transcrit d'un } \\
\text { média à un autre }\end{array}$ & $\begin{array}{l}\text { Changement de } \\
\text { référentiel ou de } \\
\text { media }\end{array}$ \\
\hline \multirow{2}{*}{ En spirale } & Elliptique & $\begin{array}{l}\text { informations chiffrées, } \\
\text { phrases sans prédicats } \\
\text { (notes, images, etc.) }\end{array}$ & $\begin{array}{l}\text { Repris comme source } \\
\text { à développer : réécrit } \\
\text { en élaborant des } \\
\text { phrases correctes } \\
\text { d'un point de } \\
\text { vue grammatical, } \\
\text { sémantique, etc. }\end{array}$ & $\begin{array}{l}\text { Transformation } \\
\text { de la mise } \\
\text { en forme, } \\
\text { organisation, } \\
\text { augmentation } \\
\text { de la taille et du } \\
\text { volume du récit }\end{array}$ \\
\hline & Prémisse & $\begin{array}{l}\text { Amorce d'un début de } \\
\text { réflexion sous forme de } \\
\text { phrases sans prédicats } \\
\text { (notes, d'images, etc.) }\end{array}$ & $\begin{array}{l}\text { Repris comme point } \\
\text { de départ d'une } \\
\text { réflexion : étoffer, } \\
\text { étayer une idée }\end{array}$ & $\begin{array}{l}\text { Transformation } \\
\text { de la mise } \\
\text { en forme, } \\
\text { organisation, } \\
\text { augmentation } \\
\text { de la taille et du } \\
\text { volume du récit }\end{array}$ \\
\hline \multirow[b]{2}{*}{ tamisage } & Pragmatique & Liste de choses à faire. & $\begin{array}{l}\text { Repris comme } \\
\text { un rappel devant } \\
\text { déclencher une action }\end{array}$ & $\begin{array}{l}\text { disparition } \\
\text { physique }\end{array}$ \\
\hline & fantômes & $\begin{array}{l}\text { Informations très } \\
\text { hétérogènes (chiffrées, } \\
\text { impressions, etc.) (notes, } \\
\text { images, etc.) }\end{array}$ & $\begin{array}{l}\text { Repris comme non } \\
\text { pertinents : évincés } \\
\text { du récit }\end{array}$ & $\begin{array}{l}\text { disparition } \\
\text { physique }\end{array}$ \\
\hline \multirow{2}{*}{$\begin{array}{l}\text { Superposition } \\
\text { Assemblage } \\
\text { montage }\end{array}$} & cumulatifs & $\begin{array}{l}\text { éléments épars } \\
\text { d'informations chiffrées } \\
\text { ou thématiques } \\
\text { éparpillées au sein d'un } \\
\text { des supports du recueil }\end{array}$ & $\begin{array}{l}\text { Repris pour être } \\
\text { regroupés, associés } \\
\text { autour d'une } \\
\text { thématique cohérente } \\
\text { ou d'un phénomène }\end{array}$ & $\begin{array}{l}\text { Réorganisation } \\
\text { spatiale }\end{array}$ \\
\hline & composites & $\begin{array}{l}\text { Informations chiffrées ou } \\
\text { thématiques éparpillées } \\
\text { au sein du recueil via des } \\
\text { médias différents }\end{array}$ & $\begin{array}{l}\text { Assemblage, } \\
\text { association et mise } \\
\text { côté à côte des ces } \\
\text { éléments divers }\end{array}$ & $\begin{array}{l}\text { Assemblage } \\
\text { multimédia }\end{array}$ \\
\hline
\end{tabular}

Tableau 3 : Typologie des instruments transitionnels

Table 3: Typology of transitional instruments

Ces instruments transitionnels mettent en évidence trois formes d'activités naratives : 
une activité linéaire, une activité en spirale et une activité de tamisage. A la lecture de ce tableau, nous pouvons noter qu'une plus ou moins grande place est laissée à l'activité de transformation au moment de l'élaboration du récit selon le type d'instruments transitionnels. Ainsi, alors que les instruments transitionnels miroirs et traduits demandent un effort minimal du narrateur, les instruments transitionnels elliptiques, prémisses et cumulatifs suscitent une implication plus importante de la part du narrateur.

En effet, les instruments miroirs peuvent correspondre à des notes brèves d'indexations spatio-temporelles du recueil qui seront reprises telles quelles au moment de l'élaboration du récit comme le montre le tableau 4 ci-dessous.

\begin{tabular}{|c|c|c|}
\hline$\frac{\text { Carnet de bord }}{\text { écrit (T2) }}$ & nool: $2, n \ldots$ & Merd) 6 Dinul \\
\hline $\begin{array}{l}\text { Document final } \\
\text { (T3) }\end{array}$ & $\begin{array}{l}\text { Mardi 30mars } \\
\text { Blablablablablabalbala } \\
\text { Blablbalabalbalabalaba } \\
\text { Balbalabalabalabalabal }\end{array}$ & $\begin{array}{l}\text { Mardi } 6 \text { avril } \\
\text { Blablablablablabalablabala } \\
\text { Blablabalabalabalablabalabla } \\
\text { blablablabalabalabalbalabalab }\end{array}$ \\
\hline
\end{tabular}

Tableau 4 : Instrument transitionnel miroir

\section{Table 4 : Mirror transitional instrument}

A contrario, les instruments transitionnels prémisses ont la particularité de se présenter en petites notes brèves au cours du déplacement, qui sont ensuite développées au cours de l'élaboration du récit. Ainsi la verbalisation suivante extraite de l'entretien d'autoconfrontation montre comment le narrateur peut noter lors de l'expédition quelques mots sans prédicats dans son carnet, et produire à partir de ces quelques mots, au cours de l'élaboration du récit, plusieurs phrases relativement longues et complexes faisant référence à ce qui s'est vécu depuis l'expédition : "Après avoir repris les éléments du carnet beige, tu ajoutes d'autres éléments? ...Oui, voilà, ... une réflexion qui vient de ce que j'ai pu lire a posteriori dans le cadre de la réflexion du colloque à XXX». En effet, les instruments transitionnels prémisses vont pousser le narrateur à approfondir la recherche d'une thématique ultérieurement, mais également les réflexions, les liens qui passent par la tête du narrateur au moment de l'élaboration du récit, lorsqu'il rencontre à nouveau les éléments recueillis. Ils font référence aux divers niveaux diégétiques ${ }^{2}$ de la narration, autrement dit à la présence conjointe dans la narration finale du personnage principal se déplaçant en fixant ses pensées, ses observations et la figure du narrateur qui donne son point de vue a posteriori.

Les instruments transitionnels cumulatifs peuvent fournir un fil rouge à la narration. Ces derniers peuvent être des instruments au cours de l'élaboration du récit pour rendre compte des différents niveaux diégétiques et notamment de l'articulation entre des éléments issus des circonstances particulières d'observation (lorsque le narrateur re-travaille des éléments du recueil) avec des énoncés généraux d'un savoir (lorsque le narrateur retravaille des informations formelles éventuellement issues de dictionnaire, encyclopédie).

Ceci, nous permet à ce stade d'apercevoir les diverses formes d'activités narratives diachroniques révélées par les instruments transitionnels à savoir des activités linéaires qui tendent à reprendre à l'identique un contenu, des activités de tamisage qui consistent à réduire, synthétiser (instruments transitionnels fantômes, traduits), les activités circulaires qui consistent à enrichir la narration (instruments elliptiques, prémisses) ainsi que les activités de montage qui consistent à assembler, effectuer un montage des divers éléments de la narration (instruments cumulatifs, pragmatiques). Par ailleurs, la typification des instruments transitionnels est relativement indépendante du contenu recueilli (ex-

2. Niveaux diégétiques : les différents niveaux de l'histoire 
emples : date, chiffre versus image versus impressions, etc.) puis repris pour l'élaboration du récit, ceci nous renvoie au caractère plurivoque des instruments transitionnels. En effet, un même contenu peut être instrumentalisé de manière différente au cours du temps, et peut alimenter l'élaboration de récits successifs et très différents.

\section{7.- Les apports de la diachronie}

\section{1.- Activités créatives et diachronie}

La diachronie apporte un éclairage heuristique. Elle permet de comprendre que les activités narratives lors du recueil entretiennent des liens serrés avec l'expérience en train de se faire, et que lors de l'élaboration du récit, celles-ci nécessitent de se reconnecter avec l'univers du déplacement. L'analyse diachronique permet donc d'examiner les mécanismes de distanciation et d'imprégnation ainsi que le rôle prépondérant du temps et des situations d'activités successives pour le narrateur. Nous proposons de discuter de deux thèmes que sont le temps et la diachronie productive, puis d'évoquer le caractère générique de ces résultats.

\section{Le temps}

Approfondissons cette notion de temporalité en convoquant Barthes. (1980). Lorsque l'auteur évoque la prise de notes, il la présente comme le découpage du flux ininterrompu de la vie et comme une nécessité (le présent colle aux yeux). Puis, Barthes avance que le texte est reproductible, matériellement grâce à l'imprimerie et phénoménologiquement par chaque lecture (à T3 ou à Tn). Ce raisonnement peut être étendu aux instruments transitionnels. Au moment de leur constitution (à T2), tantôt réductions, tantôt prélèvements, ils deviennent à T3 des instruments pour se remettre en contexte, reconstituer le contexte, manipuler la réalité, reconstruire une réalité (qu'elle soit chronologique, géographique, etc.). Elle doit être manipulable, recoupable afin d'en faire émerger des patterns. Ces fragments peuvent être repris, assemblés, redispachés et inscrits dans des productions appartenant à différentes sphères, à différents domaines d'activité (affectives, professionnelles, personnelles, esthétiques, artistiques, etc.). Pour le narrateur, réutiliser des fragments recueillis nécessite donc forcément du temps passé (un moi transformé) et une situation spatio-temporelle nouvelle, une fenêtre attentionnelle différente, plus large (autres rencontres littéraires, bibliographiques, autres rencontres des idées, etc.). A T2, les sujets travaillent leurs matériaux de diverses manières, ils peuvent s'inscrire dans des logiques diachroniques de nature différente. Ainsi certaines activités sont linéaires, en spirale, d'autres consistent à mettre de côté une partie des matériaux durant un temps (tamisage) enfin le travail de superposition tient également une place importante.

La diachronie englobe l'univers extraordinaire du déplacement et l'univers ordinaire de l'élaboration du récit, or ces situations géographiques distinctes et le temps qui s'écoule entre ces deux situations sont des ressources qui vont aider le sujet à interpréter son expérience.

\section{La diachronie productive}

Le concept d'instrument transitionnel contribue à enrichir le cadre de l'approche instrumentale. Il permet d'examiner plus particulièrement la diachronie au sein de la médiation réflexive. En effet les instruments transitionnels sont des ressources que le sujet crée pour le soi en devenir, ce sont des ressources qui permettent de créer de la continuité du côté du sujet afin de garder un lien avec l'expérience tout en pouvant la considérer comme extérieure à soi. Les instruments transitionnels permettent de penser dans le même temps des activités narratives qui sont de nature différente selon les contextes successifs : lors du recueil, les activités narratives sont synchrones et intriquées avec d'autres activités variées (rencontre de personnes, déplacements), elles permettent au narrateur de s'extraire d'une situation englobante. En revanche, elles sont au premier plan lors de l'élaboration du récit, le narrateur se retrouve en situation de visu avec ses matériaux qu'il consulte pour revisiter son expérience et construire un récit. 
Ces instruments transitionnels révèlent les diverses formes d'activités à l'œuvre au sein d'activités créatives successives ainsi que leurs réifications successives. Ils permettent de décrire les activités créatives comme un travail progressif d'extériorisations, constituées de matériaux plus ou moins matures, des ébauches en état de devenir (Meyerson, 1948).

\section{Au-delà de cette étude de cas}

Un travail plus large a été mené au sein de la thèse (Bationo-Tillon, 2006) auprès de profils variés (journalistes, blogueurs, passionnés de voyage, etc.), qui met en évidence les mêmes invariants de l'activité narrative. Cependant, cette situation d'expédition polaire nous offre de nombreux observables car c'est une situation dense qui met en exergue les dimensions spatiales et temporelles de l'activité narrative ainsi que les fonctions attribuées aux instruments transitionnels. Cette focalisation sur les activités narratives en situation extrême a donc à notre sens des vertus didactiques pour pointer des mécanismes qui comme nous en faisons l'hypothèse doivent exister chez tous les narrateurs, qu'ils soient voyageurs ou non puisque tout voyage n'a de sens que dans la mesure où il s'accomplit à l'intérieur de nous (Kassai, 1994).

\section{2.- Diachronie et conception}

De prime abord, cette recherche prospective semble relativement lointaine des problématiques de conception. Son positionnement très en amont l'isole d'un certain nombre d'implications opérationnelles ainsi que de l'inévitable créativité des futurs utilisateurs ou opérateurs tels que décrits par plusieurs auteurs (Béguin, \& Cerf, 2004 ; Folcher, 2008). En contrepartie, cette volonté d'étudier l'activité de manière diachronique nous permet de dresser un paysage étoffé des activités narratives en nous extrayant du découpage classique de la technologie. Cette recherche trace deux voies à court terme et à moyen terme, d'une part pour apporter des éléments de contribution à la conception, et d'autre part pour indiquer des directions pour penser et concevoir les technologies futures d'aide aux activités créatives.

\section{Contributions ponctuelles à différents projets}

Tout d'abord, cette analyse des activités narratives qui articule maille synchronique et maille diachronique constitue un terreau que l'ergonome peut exploiter et adresser à des acteurs de divers projets de conception. Les réductions ont lieu a posteriori, et au cas par cas (Bationo-Tillon, Genieys, $\&$ Kahn, 2007). L'analyse de l'activité selon une logique diachronique ouvre le champ des possibles pour contribuer à la conception, non pas d'un outil, mais d'un ensemble d'outils (des instruments de recueil aux instruments d'édition). Elle peut apporter des éléments à la question de l'inter-opérabilité des dispositifs, ainsi que du format et de la nature des informations qui circulent d'outils en outils (via les instruments transitionnels). Exemplifions plus précisément le type de contributions possibles. Dans le cadre d'un projet de conception de service à destination des voyageurs, nos analyses nous ont permis d'alimenter le projet tant au niveau de la définition fonctionnelle du service qu'au plan de l'élaboration du scénario d'usage (précisions sur le type de fonctionnalités relatives au carnet de voyage, ainsi qu'à la manière de les allouer sur les différents supports sur lesquels se déployait le service : téléphone mobile, site internet pour ordinateur). Pour cela nous nous sommes appuyés sur les invariants de l'activité de narration mis en évidence depuis le recueil jusqu'au partage. Nous avons également contribué à la rédaction du scénario pour le rendre cohérent et au plus proche de l'activité de récit de voyage. Par ailleurs, nous avons apporté des éléments d'éclairage dans un projet de conception de stylo communiquant afin d'aider les concepteurs à positionner cette technologie par rapport à la nature de l'activité de prise de notes lors de déplacement puis de réutilisation de ces notes au retour. Notre rôle a constitué à documenter les concepteurs sur les invariants de l'activité autour desquelles des automatisations étaient possibles (instruments transitionnels miroirs, pragmatiques) ainsi qu'à pointer les limites de ce type de dispositif au regard de la grande flexibilité de cette activité qui nécessite une part active du narrateur notamment à travers les instruments transitionnels elliptiques, prémisses. Nous les avons sensibilisés à l'hétérogénéité des supports mobilisés lors de l'étalage ainsi que de la diversité des récits potentiels à partir d'un recueil donné. 


\section{Ergonomie de prospective}

La position de l'ergonomie en amont de la conception nous semble également intéressante pour produire des recommandations plus génériques qui visent à effectuer un diagnostic entre les formes de l'activité investiguées et les types d'outils existants. C'est ce que nous nous proposons de faire ici.

Tout d'abord, le schème d'étalage est peu considéré actuellement par les outils d'aide à la narration. En effet, les espaces de consultation et de production sont majoritairement pensés, et conçus de manière disjointe. Pour outiller le schème d'étalage, il faudrait concevoir et penser les espaces de consultation et de production comme des supports de la même activité. L'espace de consultation devrait permettre de naviguer à travers une diversité de médias et privilégier la vue d'ensemble, la concomitance des éléments. Or, les outils existants ont tendance à restreindre la consultation à un écran 2D, ainsi qu'au multifenêtrage. Par ailleurs, la plupart des logiciels d'édition, de traitement et montage vidéo / photo sont spécialisés pour un type de média respectant la segmentation classique et séquentielle du recueil spécifique suivi du traitement spécifique. La navigation d'un contenu à l'autre reste donc très limitée. Peu de logiciels intègrent la complémentarité des matériaux à l'instar de Evernote qui permet de rassembler tous ses matériaux personnels qu'il s'agisse de photographies, notes, vidéo, etc.

Quant à l'espace de production, il est en général supporté par des logiciels d'édition très linéaires. Il est même inexistant dans le cas des blogues qui sont exclusivement des outils de communication. Cette activité d'élaboration mériterait donc d'être outillée. Pour cela, il faudrait s'adapter à cette activité polymorphe telle que nous l'avons décrite : linéaire, circulaire, de type tamisage, ou assemblage. Or, les artefacts numériques prédominants (ex. : logiciels d'édition) privilégient les activités linéaires, et séquentielles, au détriment du travail d'élaboration plus circulaire, plus spatial, composé de tâtonnements et de superpositions (schème d'étalage, instruments transitionnels prémisses, elliptiques) et de raffinement (instruments transitionnels fantômes). En effet, les outils d'édition actuels permettent d'effectuer des opérations de type copier, coller, supprimer, rechercher et les unités de références sont les mots ou les pages. En revanche, ils supportent peu les activités d'assemblage qui consistent à organiser le matériau, à le modeler, le réarticuler par thèmes, paragraphes. Il nous semble qu'un niveau supplémentaire serait nécessaire ainsi qu'une organisation spatiale différente, facilitant la juxtaposition. Par ailleurs remplacer la fonction de suppression par une réelle fonction de tamisage, qui ne supprime pas complètement mais consiste à mettre de côté une partie du matériau qui pourra être travaillé à nouveau dans un second temps de manière plus raffinée nous semble être une voie à explorer. Enfin, une dimension importante des activités en spirale concerne la progression de la maturité des éléments, des points d'intérêts, des formulations. Cette facette des activités narratives pourrait donc également être outillée plus spécifiquement afin d'aider le narrateur à organiser et rendre visible ce qui revient, et ce qui évolue au sein de son recueil au cours du temps.

Pour finir, l'approche diachronique de l'activité permet de révéler la quasi-absence de technologies qui s'emparent des questions d'aide à l'activité de construction, et d'élaboration narrative au cours du temps. Dans la mesure où les activités narratives et un grand nombre d'activités créatives s'inscrivent dans des temps longs, ce dernier point nous semble particulièrement problématique lorsque l'on cherche à concevoir des outils qui ne limitent pas la création.

\section{8.- Conclusion}

Considérer les activités créatives comme des activités productives diachroniques ouvre des perspectives pour revisiter certains thèmes tels que l'inspiration et la mémoire.

Tout d'abord, la diachronie permet de dépasser l'idée d'une inspiration fulgurante et propose d'étudier les activités créatives comme des activités ancrées dans l'expérience en train de se faire, puis s'élaborant par des ébauches successives. Les instruments transitionnels prémisses définis au sein de ce papier sont des fragments qui ont des caractéristiques communes avec les œuvres telles que 
décrites par Meyerson (1948). Ils fixent, résument, conservent ce qu'un homme à un moment donné a ressenti, perçu, réussi à exprimer. Ils traduisent une pensée neuve, un moment où l'esprit a tendu à progresser, à se dépasser. Ces instruments transitionnels deviennent ensuite des points d'appui et sont retravaillés, ré-assemblés, recomposés lors de l'élaboration de la narration. Nous pensons que les instruments transitionnels et le schème d'étalage mis en évidence au sein des activités narratives pourraient être présents plus largement au sein des activités créatives dans leur diversité. Cette perspective nous semble fructueuse à la lecture de Passeron (1974) qui s'est attaché à décrire l'activité des artistes peintres en train de se faire. En effet, certains peintres se déplacent pour renouveler leur inspiration en quête de nourriture artistique. Ils se promènent avec un carnet sur lequel ils fixent des traits, les croquis sont rapides souvent rattachés à un thème qui préoccupe l'artiste. A son retour à l'atelier, le peintre feuillette ses dessins, ses documents à portée de main. Il prépare l'esquisse, il tâtonne, ajoute, dose, déplace. En cours d'exécution, le peintre recule souvent de quelques pas pour mieux saisir l'ensemble de l'œuvre en train d'être créée. Les peintres ont donc également des carnets de croquis, et sont attentifs à ne pas perdre et détruire en cours de route, la fragile intégration d'un ressenti, d'un vu involontaire qui est à l'origine de l'émotion (Passeron, 1974). Ce dernier cite Corot qui rappelle qu'il ne faut jamais perdre « la première impression qui vous a ému ». Les instruments transitionnels permettraient donc de faire le lien entre les impressions, les perceptions intriquées avec l'expérience en train de se faire et l'élaboration d'une œuvre.

Pour finir, la diachronie permet également de revisiter la thématique de la mémoire qui a été largement investiguée dans le champ de la psychologie. Les diverses formes de l'activité que nous avons mis en évidence (spirales, linéaires, tamisage...) rendent compte d'une déformation de l'expérience passée par le narrateur qui la revisite en accentuant certaines facettes, en développant certains aspects et en passant sous silence d'autres dimensions. A notre sens, ces diverses formes d'activités narratives ne sont pas étrangères aux déformations mnésiques pointées par Barthes (2003). En effet, il parle d'hypermnésie pour désigner la mémoire proustienne, composée d'éclats vifs, discontinus non liés par le temps chronologique mais dont le souvenir est aigu et torrentueux. A notre sens, les récits élaborés par les narrateurs sont également des formes d'hypermnésie. Des subversions de l'expérience passée adviennent au cours de l'élaboration du récit, dans la mesure où le narrateur est engagé dans une reconstruction partielle de la réalité qu'il manipule à travers les instruments transitionnels pour en faire émerger des patterns chronologiques ou thématiques.

\section{RÉFÉRENCES}

Ackermann, E. (2008). Notation chez l'enfant : Du Graphique au numérique. In D. Andler, \& B. Guerry, (Eds.), Apprendre demain : Sciences cognitives et éducation à l'ère numérique (pp. 77-94). Paris : Hatier (Coll. Cap Digital Education. Groupe Compas).

Agostini, A., De Michelis, G., Divitini, M., Grasso, M.-A., \& Snowdon, D. (2002). Design and deployment of community systems : reflections on the Campiello experience. Interacting with Computers, 14, 689712 .

Barriquault, C. (2005). Représentation d'incidents dans le retour d'expérience de la navigation aérienne. Doctorat de psychologie, Saint Denis: Université Paris 8.

Barthes, R. (1980). La chambre claire : notes sur la photographie. Pais: Seuil.

Barthes, R. (2003). La préparation du roman I et II : Cours et séminaires au Collège de France (1978-1979 et 19779-1980). Paris: Seuil.

Bationo Tillon, A. (2006) Pratiques des activités narratives instrumentées: Une analyse diachronique et structuro-fonctionnelle en amont de la conception. Doctorat d'ergonomie, Saint Denis: Université de Paris 8 .

Bationo Tillon, A., Genieyes, G., \& Kahn, J.(2007) Quelle place pour l'analyse de l'activité en situation naturelle dans un cycle de conception de produit grand public ? In M. Zouinar, G. Valléry, \& M.C. Le 
Port, (Eds.), 42ème congrès de Société d'ergonomie de Langue française : Ergonomie des produits et des services (pp 537-548). Saint Malo: Octarès Editions.

Beaudouin, V., \& Velkovska, J. (1999). Constitution d'un espace de communication sur internet. Réseaux, 97, 121-177.

Béguin, P., \& Cerf, M. (2004). Formes et enjeux de l'analyse de l'activité pour la conception des systèmes de travail. Activités, 1(1), 54-71.

Bordwell, D. (1985). Narration in fiction film. London: Routledge.

Bourdieu, P. (1965). Un art Moyen, essai sur les usages sociaux de la photographie. Paris: Les éditions de Minuit.

Bouvier, N. (1992). Usage du monde. Paris: Payot.

Bruner, J.-S. (1990). Acts of meanings. Cambridge: Havard University Press.

Cardon, D., \& Delaunay-Teterel, H. (2006). La production de soi comme technique relationnelle. Un essai de typologie des blogs par leurs publics. Réseaux, 138, 90-110.

Decortis, F., Daele, L., Polazzi, L., Rizzo, A., \& Saudelli, B. (2001). Nouveaux instruments actifs et activités narratives. Revue d'interactions hommes-machines, 2 N2/2001, 1-30.

Dodier, N. (1990). Représenter ses actions : le cas des inspecteurs et des médecins du travail. In P. Pharo, \& L. Quéré (Eds.), Les formes de l'action (pp. 115-148). Paris: Raisons pratiques

Eco, U. (1985). Lector in Fabula. Paris: Grasset.

Folcher, V. (2008). Conception pour l'usage - conception dans l'usage : propositions pour une rencontre. In I. Bloch, \& F. Conne (Eds.), Actes de la XIVème Ecole d'été de Didactique des mathématiques (pp. 1-13). Paris: La Pensée Sauvage Editions.

Glos, J.-W, \& Cassell, J. (1997). Rosebud: A Place for Interaction Between Memory, Story, and Self. Proceedings of the 2nd International Conference on Cognitive Technology (CT'97), 88 (pp. 359-360). Atlanta Georgia Conference on Human Factors in Computing Systems.

Kassai, G. (1994). Elaboration d'un récit de voyage. In G. Tverdota (Ed.), Ecrire le voyage (pp 241-246). Paris: Presses de la Sorbonne nouvelle.

Laplantine, F. (1996). La description ethnographique. Paris: Nathan.

Latour, B. (1996). Petites leçons de sociologie des sciences. Paris: La Découverte.

Lebahar, J.-C. (1983). Le dessin d'architecte : simulation graphique et réduction de l'incertitude. Marseille: Parenthèse.

Lejeune, P. (1998). Les brouillons de soi. Paris: Le Seuil.

McCloud, S. (1993). Understanding comics the invisible art. New York: Harper Collins.

Meyerson, I. (1948). Les fonctions psychologiques et les æuvves. Paris: Vrin.

Orr, J. (1996). Talking About Machines: An ethnography of a modern job. Itacha, NY : Cornelle University Press

Passeron, J. C. (1974). L'œuvre picturale et les fonctions de l'apparence. Paris: Vrin.

Piaget, J. (1974). Réussir et comprendre. Paris: PUF.

Pinault, M. (1994). L'écriture du peintre Jean-Pierre Laurent Houel. In G. Tverdota (Ed.), Ecrire le voyage (pp. 69-80). Paris: Presses de la Sorbonne nouvelle.

Rabardel, P. (1995). Les hommes et les technologies, approche cognitive des instruments contemporains. Paris: Armand Colin.

Rabardel, P. (2001). Instrument mediated activity in situations. In A. Blandford \& P. Vanderdonckt (Eds.), People and computers XV Interactions without frontiers (pp. 17-30). Verlag: Springer. 
Rix, G., \& Biache, M-J. (2004). Enregistrement en perspective subjective située et entretien en resitu subjectif : une méthodologie de constitution de l'expérience. Intellectica, n³8, 363-396.

Soulier E. (2006). Le storytelling : concepts, outils et applications. Paris: Hermès sciences, Lavoisiers.

Theureau, J. (2004). Cours d'action : méthode élémentaire. Toulouse: Octarès Editions.

Umaschi, M. (1996). SAGE Storytellers: Learning about Identity, Language and Technology. Proceedings of ICLS '96 , AACE, pp. 526-531.

Vergnaud, G. (1985). Concepts et schèmes dans la théorie opératoire de la représentation. In S. Ehrlich (Ed.), Les représentations, 30, 3-4 (pp. 245-252). Paris: Psychologie française.

Vermersch, P. (1994). L'entretien d'explicitation. Issy Les moulineaux: ESF.

West, D., Quigley, A., \& Kay, J. (2007). MEMENTO: A digital-physical scrapbook for memory sharing. Personal and Ubiquitous Computing, 11(4), 313-328.

Vygotsky, L.-S. (1934). Pensée et langage. Paris: Editions sociales.

Vygotsky, L.-S. (1973). Immaginazione e creativa nell' età infantile. Rome: Riunti.

Winnicott, D.-W. (1971). Objet transitionnels et phénomènes transitionnels, Jeu et réalité. Paris: Gallimard.

\section{RÉSUMÉ}

Cet article présente les activités narratives de deux expéditeurs polaires. Nous adoptons une unité d'analyse diachronique qui englobe la constitution du recueil lors du voyage ainsi que l'élaboration du récit après le voyage. Dans cette optique, nous articulons théoriquement et méthodologiquement l'approche instrumentale et le cours d'action. Nous proposons le concept d'instrument transitionnel pour désigner ce qui transite au sein de ces activités narratives discontinues. Lors du voyage, les narrateurs opèrent une mise à distance de l'expérience grâce aux activités de recueil instrumentées (prises de notes, de photographies). C'est à ce moment là qu'ils constituent leurs instruments transitionnels. Après le voyage, les narrateurs se réimprègnent de l'expérience de voyage grâce à l'étalage des éléments recueillis (croquis, cartes, écrits..). Ils élaborent le récit en assemblant les instruments transitionnels. Pour finir, nous discutons des apports de la diachronie pour mieux étudier et outiller les activités créatives.

\section{MotS CLÉS}

activités narratives, activités créatives, activités diachroniques, approche instrumentale, cours d'action

\section{RÉFÉRENCEMENT}

Bationo Tillon, A., Folcher, V., \& Rabardel, P. (2010). Les artefacts transitionnels: une proposition pour étudier la diachronie des activités narratives. Activités, 7(2), 63-83. http://www.activites.org/v7n2/v7n2. pdf

Article soumis le 9 septembre 2009, accepté pour publication le 30 mars 2010. 